\title{
LES GÉNÉRATIONS REDIENNES DE FASCIOLA HEPATICA L. CHEZ LYMNAEA TRUNCATULA MÜLLER
}

\section{à propos des effets de plusieurs facteurs}

\author{
D. RONDELAUD et D. BARTHE*
}

\begin{abstract}
RÉSUMÉ. Lymnaea truncatula infestée par Fasciola hepatica présente une succession de trois générations rédiennes lorsque des mollusques de $4 \mathrm{~mm}$ de hauteur sont exposés chacun à un seul miracidium et élevés ensuite à $20^{\circ} \mathrm{C}$ jusqu'au $30^{\mathrm{e}}$ jour post-exposition. L'origine géographique de $L$. truncatula ne retentit que sur le nombre de rédies des générations 2 et 3 . L'origine géographique des miracidiums n'a pas d'influence sur le développement de ces générations rédiennes. La succession de ces générations est par contre modifiée chez les mollusques soumis à des infestations plurimiracidiennes. A partir de 5 miracidiums pour une seule exposition, s'observe une chute du nombre moyen des rédies affectant surtout les générations 2 et 3 ; pour les rédies des générations 1 et 2, il existe un retard de maturation d'autant plus grand que le nombre de miracidiums est plus élevé. A partir de 4 expositions monomiracidiennes successives, on retrouve les résultats précités.

La signification de ces observations est discutée.
\end{abstract}

\section{The effects of some factors on the redial generations of Fasciola hepatica $\mathrm{L}$. in Lymnaea truncatula Müller}

SUMMARY. There was a succession of 3 redial generations in 4-mm-high snails individually exposed to a single miracidium and then bred at $20^{\circ} \mathrm{C}$ during 30 days after exposure. The geographic strain of Lymnaea truncatula only affected the number of rediae of generations 2 and 3. The geographic strain of miracidia of Fasciola hepatica had no influence on the development of these redial generations.

The succession of these redial generations changed in the case of plurimiracidial exposures. In snails once exposed to high numbers of miracidia (from 5 to 20), the mean number of rediae per snail decreased affecting especially the generations 2 and 3 ; the maturation of rediae of generations 1 and 2 was later when the number of used miracidia was higher. The same results were found in snails individually subjected to 4 exposures with one miracidium per exposure. The significance of these observations is discussed.

* Laboratoire d'Histologie-Embryologie-Cytogénétique, Faculté de Médecine, 2, Rue du Docteur Marcland, F 87032 Limoges Cedex.

Accepté le r9 octobre r98I. 


\section{Introduction}

On admet depuis les travaux de Leuckart $(1882,1883)$ et de Thomas (1883) que chaque sporocyste donne naissance à une première génération de rédies appelées encore rédies mères. Ces dernières peuvent, soit former directement des cercaires, soit donner naissance à une deuxième génération de rédies (rédies filles), ces dernières formant elles-même les cercaires. L'existences de ces rédies est encore débattue à l'heure actuelle : leur production serait contrôlée par la saison (Leuckart, 1882, 1883 ; Thomas, 1883), par les expositions aux basses températures (Kendall, 1964, 1965) ou par le jeûne du mollusque (Styczynska-Jurewicz, 1965) ; Wilson et Draskau (1976) confirment que la production de ces rédies filles est stimulée lorsque le mollusque hôte est soumis pendant des temps variables à l'influence de températures chaudes ou froides ou encore au jeûne après l'exposition aux miracidiums.

L'étude histologique de limnées tronquées (Lymnaea truncatula) récoltées infestées sur le terrain (Rondelaud, 1974 a) ou exposées aux miracidiums de Fasciola hepatica dans les conditions du laboratoire (Rondelaud, 1978; Rondelaud et Barthe, 1978 a) montre que le schéma de développement des formes larvaires de ce parasite est en réalité plus complexe. Plusieurs générations rédiennes s'observent chez les limnées lorsqu'elles sont exposése chacune à un seul miracidium :

- Chaque sporocyste produit des rédies de génération 1.

- La première rédie issue du sporocyste (rédie no 1 ) se développe rapidement dans le corps du mollusque hôte et forme des rédies de génération 2 en nombre élevé (rédies précoces). L'émergence de ces rédies du corps de la rédie mère est progressive et synchrone de celle des autres rédies de génération 1 à partir du sporocyste.

Les autres rédies de génération 1 forment de même des rédies en même temps que les cercaires. Ces rédies filles peu nombreuses et plus tardives font partie également de la génération 2 (rédies tardives).

- Les rédies précoces de la génération 2 produisent des rédies de génération 3 en même temps que les cercaires. Leur émergence est synchrone de celle des rédies tardives de génération 2 et survient après le $35^{\mathrm{e}}$ jour post-exposition à $20^{\circ} \mathrm{C}$.

Ce schéma de développement est valable jusqu'à l'apparition des premières cercaires indépendantes dans le corps du mollusque hôte, soit durant les 49 premiers jours post-exposition à $20^{\circ} \mathrm{C}$.

Une première étude expérimentale montre que cette succession de générations rédiennes est toujours présente chez les limnées tronquées élevées en fonction de divers facteurs étudiés isolément : température d'élevage, dessèchement du milieu de vie du mollusque, volume corporel du mollusque hôte (Rondelaud et Barthe, 1978 a). 
Nous nous proposons dans la présente note :

- d'analyser les effets de 4 autres facteurs (précisés ci-après) sur la dynamique de ces générations rédiennes chez des limnées sacrifiées toutes au $30^{\mathrm{e}}$ jour post-exposition à $20^{\circ} \mathrm{C}$;

— de compléter le schéma d'évolution de ces générations (déjà publié : Rondelaud et Barthe, $1978 a$ ) en précisant le nombre moyen de rédies qui se développent à partir d'un seul sporocyste.

\section{Matériel et méthodes}

\section{1 - Les séries expérimentales.}

Quinze séries ont été constituées. Les caractéristiques des limnées infestées ou témoins de chaque série sont précisées sur le tableau $I$.

Ces séries expérimentales ont été constituées en vue d'étudier les effets de 4 facteurs pris isolément :

1 - l'origine géographique de la population de limnées. Trois populations vivant sur terrain siliceux (département de la Haute-Vienne : séries 1 à 3 ) et trois autres populations vivant sur terrain calcaire (départements de l'Indre et de la Vienne : séries 4 à 6) ont été retenues dans le cadre de ce travail. La vitesse de croissance des limnées de chaque population est fonction de la nature géologique du sédiment sur lequel les mollusques vivent : au $30^{\mathrm{e}}$ jour post-exposition, les limnées infestées provenant de terrains calcaires ont une hauteur de coquille plus élevée que celle des limnées de terrains siliceux $(9,75$ à $10,76 \mathrm{~mm}$ contre 8,02 à $9,23 \mathrm{~mm}$ respectivement : tableau I).

2 - l'origine géographique des miracidiums de Fasciola. Seuls des œufs provenant de bovins fortement infestés par la fasciolose ont été utilisés. L'origine géographique de ces bovins diffère : région de Bellac (Haute-Vienne) pour la série 1, région de Parthenay (Deux-Sèvres) pour la série 7 et région de Poitiers (Vienne) pour la série 8.

3 - le nombre de miracidiums présents pour une exposition. Ce nombre varie de 1 à 20 miracidiums (séries $1,9,10,11$ et 12).

4 - le nombre d'expositions monomiracidiennes. Chaque mollusque est exposé tous les deux jours à un miracidium isolé et ceci 2, 3 ou 4 fois (séries 1, 13, 14 et 15).

$\mathrm{Au} 30^{\mathrm{e}}$ jour qui suit la première exposition aux miracidiums, tous les mollusques survivants sont sacrifiés après mesure de la hauteur de la coquille. Il en est de même pour les limnées témoins. 
D. RONDELAUD ET D. BARTHE

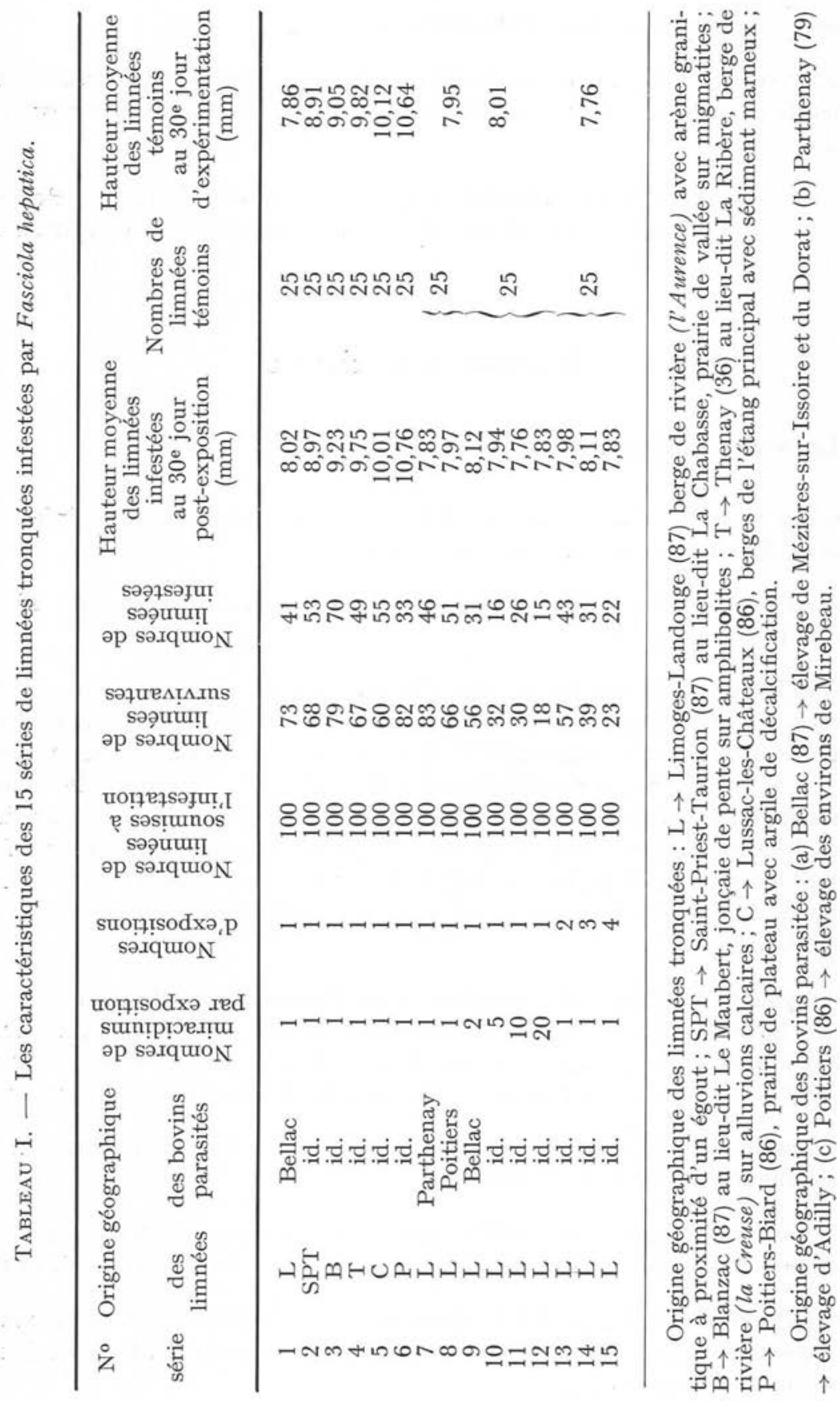




\section{2 - Les méthodes utilisées.}

\section{A) Technique d'élevage.}

Des bacs d'élevage standard de limnées (Rondelaud, 1974 b) ont été constitués en utilisant le sédiment et l'eau des habitats naturels de chaque population intéressée par cette étude. L'eau est maintenue à niveau constant, oxygénée artificiellement et renouvelée partiellement 2 fois par semaine. Les autres conditions d'élevage sont standard : température de $20^{\circ} \pm 1^{\circ} \mathrm{C}$, photophase diurne de 12 heures avec une intensité de 3000 lux par bac. Des fragments de salade en lyse sont ajoutés deux fois par semaine en complément de la nourriture algale présente dans les bacs.

Après leur récolte, les limnées mesurant alors $4 \pm 0,1 \mathrm{~mm}$ de hauteur sont laissées pendant 8 jours en stabulation au laboratoire à raison de 50 individus par bac.

\section{B) Technique d'infestation.}

Les œufs de Fasciola hepatica sont recueillis à l'abattoir local dans la bile provenant de la vésicule biliaire de bovins. Après lavage, les œufs sont laissés à l'obscurité pendant 20 jours à $20^{\circ} \mathrm{C}$ (Ollerenshaw, 1971).

Chaque limnée est exposée à un ou plusieurs miracidiums juste éclos pendant 6 heures environ jusqu'à l'apparition de deux signes : la disparition du ou des miracidium(s) du récipient d'expérience et la rétraction du mollusque dans sa coquille avec émission de mucus.

\section{C) Technique histologique.}

Les limnées destinées au sacrifice sont plongées directement dans du Bouin et leurs coquilles sont rapidement détruites sous loupe binoculaire de manière à laisser en place les parthenitae. Les animaux sont ensuite débités en coupes sériées de $5 \mu \mathrm{m}$ d'épaisseur et colorés par l'hématoxyline de Harris - trichrome de Gabe modifié.

\section{3 - Le classement des parasites.}

Les divers parasites présents sur les coupes histologiques sont classés en fonction de plusieurs paramètres :

A) La morphologie des pharynx des rédies.

Les rédies de génération 1 présentent un pharynx sphérique avec une lumière interne large, généralement ronde, parfois ovoïde. Cette caractéristique structurale 
s'observe chez les jeunes rédies encore contenues dans le sporocyste et chez les rédies indépendantes (Rondelaud, $1974 a$; Rondelaud et Barthe, $1978 a$ ).

Les rédies des générations ultérieures (2 et 3) ont par contre un pharynx ovoïde, parfois sphérique avec une lumière toujours étroite chez les rédies jeunes et devenant un peu plus ovoïde dans la partie basale du pharynx chez les rédies plus âgées.

Les rédies des générations 2 ou 3 ne peuvent donc être donc identifiées d'après les seules caractéristiques des pharynx. C'est la raison pour laquelle notre étude porte sur des limnées sacrifiées toutes au $30^{\circ}$ jour post-exposition à $20^{\circ} \mathrm{C}:$ les rédies tardives de génération 2 et les rédies de génération 3 sont alors contenues dans le corps de leurs rédies mères respectives, ce qui permet d'individualiser facilement les trois générations rédiennes.

B) Les stades parasitaires des rédies.

Le stade parasitaire d'une rédie (Rondelaud, 1974 a) désigne l'état d'évolution de l'embryon le plus mature situé dans cette rédie. Nous avons utilisé les stades suivants : (a) stade 1 (morula sphérique); (b) stade 2 a (morula ovoïde avec intestin ébauché) ; (c) stade $2 \mathrm{~b}$ (morula plus aplatie avec prolongement caudal reconnaissable) ; (d) stade 3 (cercaire en formation avec cellules cystigènes non sécrétantes) ; (e) stade 4 (cercaire présentant une couche protectrice externe).

C) L'état physiologique des parasites.

Dans le cas des sporocystes, nous ne considérons que deux catégories selon qu'ils soient en vie ou en dégénérescence.

Pour chaque génération de rédies, nous tenons compte des catégories suivantes : (a) rédies encore contenues dans les sporocystes ou dans d'autres rédies (rédies dépendantes) ; (b) rédies libres dans le corps du mollusque, soit en vie (rédies indépendantes en vie), soit en dégénérescence (rédies indépendantes en dégénérescence).

Les rédies contenues dans un sporocyste ou une rédie en dégénérescence n'ont pas été retenues dans ce décompte.

Sous ce terme de dégénérescence (Rondelaud et Barthe, 1980), sont rassemblés des parasites qui involuent en présentant des noyaux pycnotiques ou en lyse de même que certains pharynx rédiens entourés de " tissu fibreux ".

Quelques rédies contenues dans les parois de la glande hermaphrodite ou dépourvues de masses germinatives (Rondelaud et Barthe, 1980) ont été comptées dans les rédies indépendantes en vie.

\section{4 - Expression des résultats.}

Les résultats de 13 séries expérimentales sont indiqués sur les figures 1, 2 et 3. Ces données se rapportent aux nombres moyens de parasites établis pour chaque 
série en tenant compte : (a) de leur nature (sporocyste ou rédie), (b) de leur état physiologique, (c) de la génération concernée pour les rédies.

Dans un but de clarification, les valeurs extrêmes n'ont été précisées que pour les nombres totaux de sporocystes ou de rédies contenus dans un mollusque

Les commentaires de chaque figure portent successivement : (a) sur les sporocystes présents (sauf pour la figure 1), (b) sur les rédies classées seulement par catégorie, (c) sur ces mêmes rédies en tenant compte de leur génération et de leur état physiologique.

\section{Résultats}

Les limnées tronquées sacrifiées au $30^{\mathrm{e}}$ jour post-exposition contiennent des rédies indépendantes se rapportant aux générations 1 et 2 . Les sporocystes ont été retrouvés chez $75 \%$ des limnées pour les 8 premières séries expérimentales; par contre les mollusques des 7 autres séries (9 à 15) en présentent toujours en nombre variable.

Les rédies tardives de génération 2 et les rédies de génération 3 sont encore contenues dans le corps de leurs rédies mères respectives.

1 - L'origine géographique des limnées d'expérimentation (séries 1 à $6:$ fig. 1).

$\mathrm{Au} 30^{\circ}$ jour post-exposition, les limnées de la série 1 (Limoges) présentent en moyenne 22 rédies indépendantes par individu dont 4 en dégénérescence (fig. $1 \mathrm{~A}$ et $1 \mathrm{~B}$ ) ; de plús 7 rédies sont encore dépendantes dans le sporocyste ou les rédies précédentes (fig. $1 \mathrm{C}$ ).

Pour les limnées des 5 autres séries, le nombre de rédies indépendantes en vie est sensiblement identique à celui de la série 1 ( fig. $1 \mathrm{~A}$ ). En revanche on assiste à une diminution du nombre moyen des rédies indépendantes en dégénérescence en allant de la série 2 à la série 6 (fig. $1 \mathrm{~B}$ ) tandis que les rédies encore dépendantes augmentent en nombre (fig. 1 C).

La distribution de ces rédies en fonction de leur génération et de leur état physiologique est indiquée sur les figures $1 \mathrm{D} \grave{a} 1 \mathrm{~K}$ :

a - génération 1. Le nombre moyen de rédies indépendantes en vie est sensiblement identique pour les limnées des 6 séries $(6,2$ à 7,1 rédies en moyenne par individu : fig. $1 D)$; il en est de même pour les rédies encore présentes dans le sporocyste $(1,2$ à 1,9 rédies par individu : $f i g .1 F)$. En revanche le nombre de rédies indépendantes et en dégénérescence est plus faible pour les séries 4 à $6(0,7$ à 1,4 rédies en moyenne par individu : fig. $1 \mathrm{E}$ ) que pour les trois premières séries (2 à 2,4 rédies par individu). 

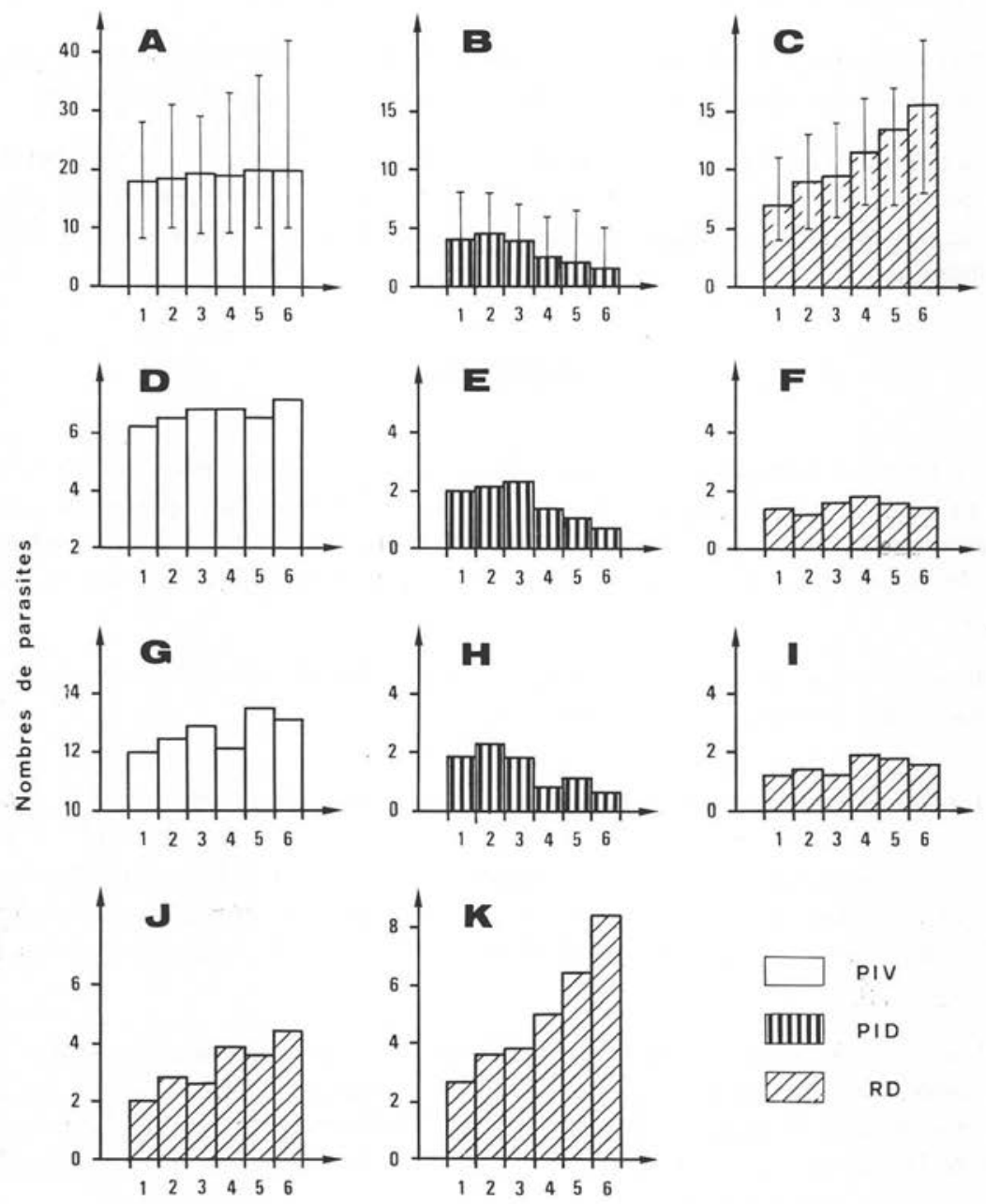

\section{Numéro d'ordre des séries expérimentales}

FIG. I. - Les nombres moyens de rédies comptées au $30^{\mathrm{e}}$ jour post-exposition chez les limnées des séries I à 6: (a) Les rédies de toutes les générations (fig. I A à I C); (b) Les rédies de génération I (fig. I D à I F) ; (c) Les rédies précoces de génération 2 (fig. I G à I I) ; (d) Les rédies tardives de génération 2 (fig. I J) ; (e) Les rédies de génération 3 (fig. I K).

Rédies indépendantes en vie (PIV) ou en dégénérescence (PID) ; rédies encore dépendantes (RD), soit du sporocyste (fig. I F), soit de la $I^{\text {re }}$ rédie de génération $\mathrm{I}$ (fig. I I), soit des autres rédies de générations I (fig. I J) ou 2 (fig. I K).

Les valeurs extrêmes ne sont indiquées que sur les figures I $\mathrm{A}$ à I C. 
b - génération 2. Les résultats concernant les rédies précoces sont comparables à ceux obtenus pour les rédies de génération 1 : les rédies indépendantes en vie (fig. $1 \mathrm{G}$ ) ainsi que celles encore situées dans le corps de la rédie no 1 de génération 1 ( fig. 1 I) sont sensiblement équivalentes en nombres pour les limnées des 6 séries; les rédies indépendantes en dégénérescence présentent des nombres plus faibles pour les séries 4 à 6 ( 0,6 à 1,1 rédies en moyenne par individu : fig. 1 H) que pour les trois premières séries ( 1,8 à 2,3 rédies par limnée).

Le nombre de rédies tardives encore dépendantes des autres rédies de génération 1 s'accroît en allant de la série 1 à la série 6 ( fig. $1 \mathrm{~J}$ ).

c - génération 3 ( fig. $1 \mathrm{~K}$ ) : on note également un accroissement marqué du nombre de ces rédies en allant de la série 1 à la série 6 .

La plupart des rédies indépendantes chez les limnées de ces 6 séries présentent le même stade parasitaire no 3 (résultats non représentés).

2 - L'origine géographique des miracidiums (séries 1,7 et 8 : résultats non représentés).

Les trois souches de miracidiums proviennent de bovins originaires de trois régions différentes.

Les résultats obtenus chez les limnées de la série 7 se superposent à ceux fournis par l'examen des mollusques de la série 1 . Il en est de même pour les limnées de la série 8, mais la dispersion des résultats concernant les rédies indépendantes et dépendantes est plus grande allant respectivement de 2 à 39 rédies pour les premières et de 2 à 13 pour les secondes (valeurs moyennes respectives : 31,2 et 7,2 rédies par individu).

Les stades parasitaires des rédies indépendantes en vie chez les mollusques des séries 7 et 8 sont identiques à ceux des rédies chez les limnées de la série 1.

3 - Le nombre de miracidiums par exposition (séries 1 et 9 à $12:$ fig. 2).

L'augmentation du nombre de miracidiums s'accompagne au $30 \mathrm{e}$ jour postexposition : a) d'un accroissement peu marqué du nombre de sporocystes dans la limnée (3,1 et 5,6 sporocystes respectivement en vie et en dégénérescence en moyenne par limnée dans la série 12 : fig. $2 \mathrm{~A}$ ) ; b) d'une diminution du nombre moyen de rédies indépendantes en vie (de 18 rédies en moyenne par limnée pour la série 1 à 13,2 rédies en moyenne par individu pour la série 12) malgré la dispersion des résultats (fig. $2 \mathrm{~B}$ ); c) d'une diminution du nombre moyen de rédies indépendantes en dégénérescence (de 4 rédies en moyenne par limnée pour la série 1 à 2 rédies par individu pour la série 12 : fig. $2 \mathrm{~B}$ ); d) d'une augmentation du nombre de rédies encore dépendantes des sporocystes ou des autres rédies (fig. $2 \mathrm{~B}$ ). 

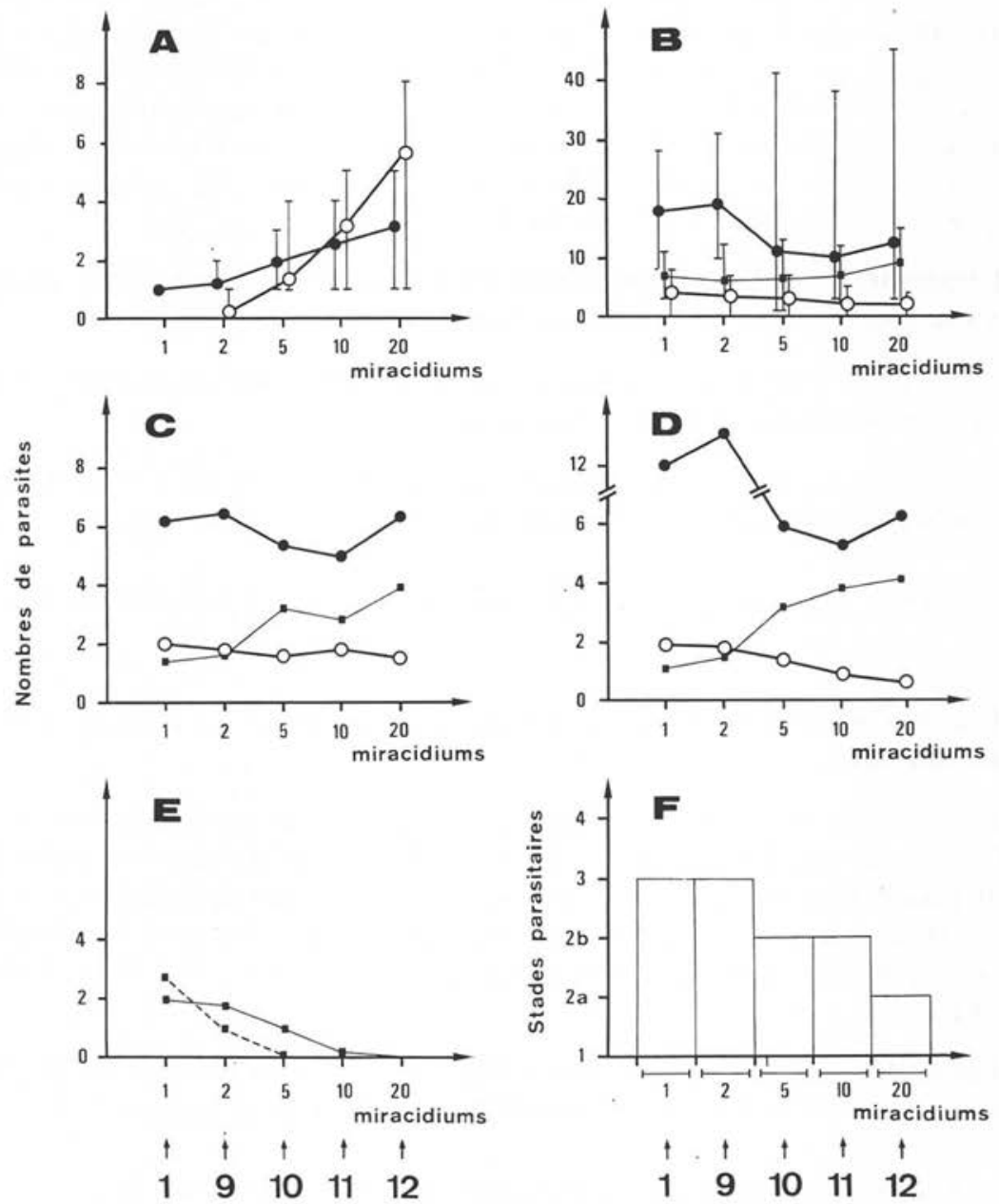

Numéro dordre des séries expèrimentales

- PIV O PID : RD

FIG. 2. - Les nombres moyens de parasites et leurs stades parasitaires au $30^{\mathrm{e}}$ jour post-exposition chez les limnées des séries 1 et 9 à 12 : (a) Les sporocystes (fig. 2 A) ; (b) Les rédies de toutes les générations (fig. 2 B); (c) Les rédies de génération I (fig. 2 C); (d) Les rédies précoces de génération 2 (fig. 2 D) ; (e) Les rédies tardives de génération 2 (trait continu) et les rédies de génération 3 (pointillés : fig. $2 \mathrm{E}$ ); (f) Les stades parasitaires des rédies les plus matures (fig. 2 F).

Parasites indépendants en vie (PIV) ou en dégénérescence (PID) ; rédies encore dépendantes (RD), soit des sporocystes (fig. 2 C), soit des premières rédies de génération I (fig.2 D), soit des autres rédies de générations I et 2 (fig. 2 E).

Les valeurs extrêmes ne sont indiquées que sur les figures $2 \mathrm{~A}$ et $2 \mathrm{~B}$. 
Les figures $2 C \grave{a} 2 E$ indiquent la distribution des rédies en fonction de leur génération et de leur état physiologique :

a - génération 1 ( fig. $2 \mathrm{C}$ ). Les nombres moyens de rédies indépendantes en vie et en dégénérescence varient peu, quelle que soit la série expérimentale (respectivement de 5 à 6,4 rédies et de 1,6 à 2 rédies par mollusque). En revanche le nombre de rédies encore contenues dans les sporocystes en vie croît avec le nombre de miracidiums atteignant une valeur de 4,1 rédies par limnée dans la série 12 . De même il faut noter un accroissement du nombre des rédies no 1 de génération 1 (de 1 rédie par limnée pour les séries 1 et 9 à 2,7 rédies par individu pour la série 12 : résultats non représentés).

b - génération 2 (fig. $2 \mathrm{D}$ et $2 \mathrm{E}$ ). A partir de 5 miracidiums (série 10), le nombre de rédies précoces indépendantes et en vie diminue de moitié par rapport à celui noté pour les séries 1 et 9 pour venir se stabiliser à 5-6 rédies par mollusque. Par contre le nombre moyen de rédies encore dépendantes des rédies $n^{0} 1$ augmente pour atteindre 3-4 rédies par mollusque. Le nombre moyen de rédies précoces indépendantes et en dégénérescence diminue progressivement de la série 1 à la série 12 (fig. 2 D).

On assiste à une diminution graduelle du nombre des rédies tardives, puis à leur disparition au fur et à mesure que le nombre de miracidiums croît (fig. 2 E).

c - génération 3 (fig. 2 E) : ces rédies disparaissent à partir de la série 10 .

Sur la figure $2 \mathrm{~F}$, sont indiqués les stades parasitaires des rédies les plus matures observés chez les limnées des diverses séries au $30^{\circ}$ jour post-exposition. On note un retard de maturation d'autant plus grand que le nombre de miracidiums présents par exposition est plus élevé.

4 - Le nombre d'expositions aux miracidiums (séries 1 et 13 à $15:$ fig. 3).

Si la limnée est exposée plusieurs fois de suite à un miracidium, le nombre de sporocystes en vie au $30^{\mathrm{e}}$ jour post-exposition est élevé $(3,1$ en moyenne par limnée : série 15) tandis que celui des sporocytes en dégénérescence est faible $(0,4$ en moyenne pour la même série : fig. $3 \mathrm{~A}$ ). Les nombres des rédies indépendantes ou non sont respectivement semblables pour toutes les séries (fig. $3 \mathrm{~B}$ ).

La distribution de ces rédies en fonction de leur génération et de leur état nhvsiologique est précisée sur les figures $3 C$ à $3 E$ :

a - génération 1 ( fig. $3 C$ ) : les rédies encore dépendantes des sporocystes augmentent très nettement en nombre avec le nombre d'expositions ( 5,5 rédies en moyenne par individu pour la série 15). Le nombre de rédies indépendantes en vie est par contre sensiblement identique. On note une augmentation des nombres moyens de rédies no 1 dans les séries 13 à 15 (respectivement de 1,5 à 2,4 rédies en moyenne par individu : résultats non représentés). 

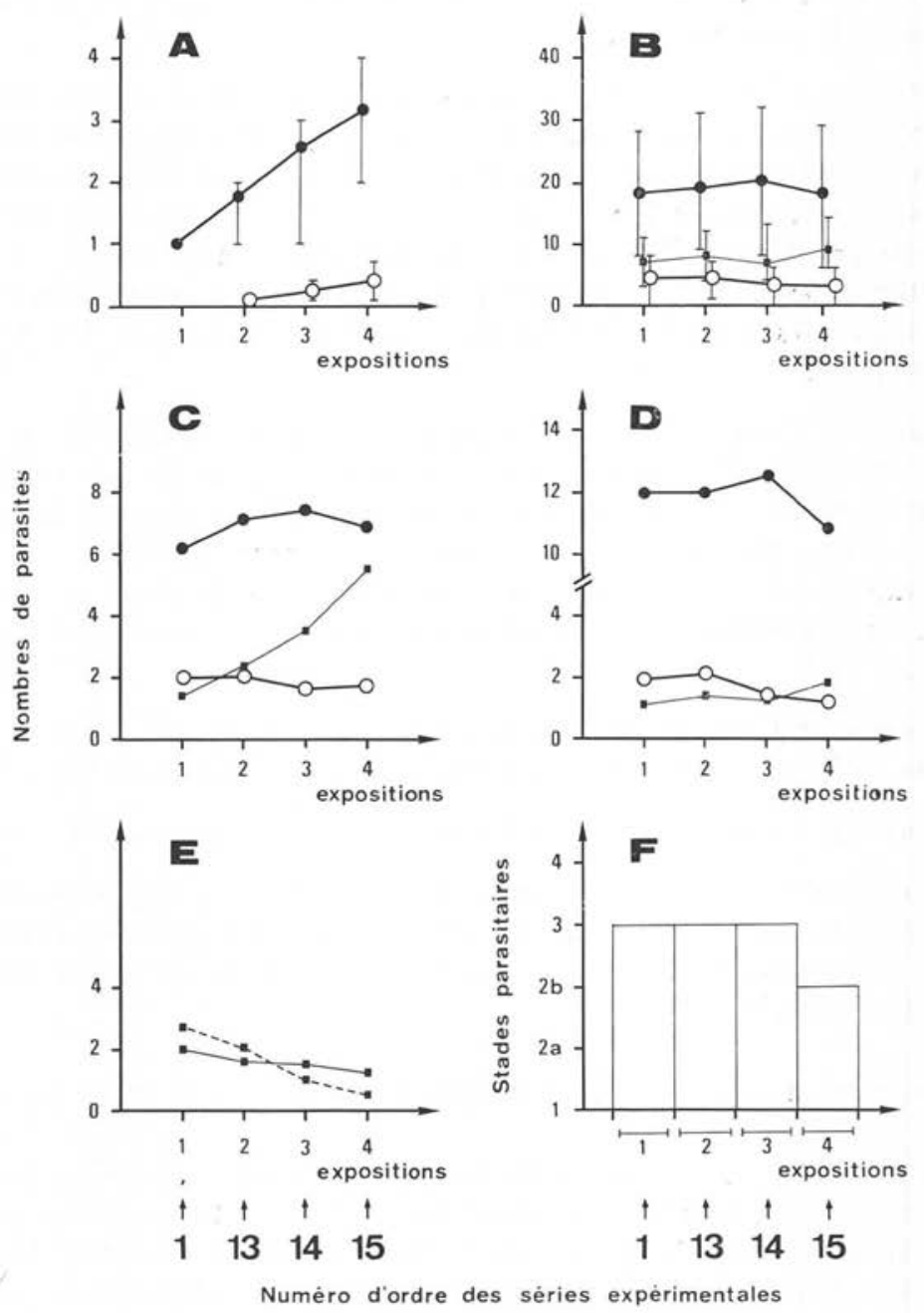

- PIV O PID . RD

FIG. 3. - Les nombres moyens de parasites et leurs stades parasitaires au $30^{\mathrm{e}}$ jour post-exposition chez les limnées des séries I et $I_{3}$ à $I_{5}$ : (a) Les sporocystes (fig. 3 A); (b) Les rédies de toutes les générations (fig. $3 \mathrm{~B}$ ) ; (c) Les rédies de génération I (fig. $3 \mathrm{C}$ ) ; (d) Les rédies précoces de génération 2 (fig. 3 D) (e) Les rédies tardives de génération 2 (trait continu) et les rédies de génération 3 (pointillés : fig. $3 \mathrm{E}$ ); (f) Les stades parasitaires des rédies les plus matures (fig. 3 F).

Parasites indépendants en vie (PIV) ou en dégénérescence (PID) ; rédies encore dépendantes (RD), soit des sporocystes (fig. $3 \mathrm{C}$ ), soit des rédies $\mathrm{n}^{0} \mathrm{I}$ de génération $\mathrm{I}$ (fig. $3 \mathrm{D}$ ), soit des autres rédies de générations I et 2 (fig. 3 E).

Les valeurs extrêmes ne sont indiquées que sur les figures $3 \mathrm{~A}$ et $3 \mathrm{~B}$. 
Les rédies indépendantes en dégénérescence sont en nombre constant, quelle que soit la série expérimentale.

b - génération 2 (fig. $3 D$ et $3 E$ ). Chaque catégorie de rédies précoces présente des chiffres qui fluctuent peu, malgré le nombre d'expositions. On note surtout une légère chute du nombre moyen des rédies indépendantes en vie à partir de la $4 \mathrm{e}$ exposition (fig. $3 \mathrm{D}$ ).

Le nombre de rédies tardives diminue graduellement au fur et à mesure des expositions ( $f i g .3 \mathrm{E}$ ).

c - génération 3 (fig. 3 E) : il existe une diminution marquée du nombre moyen de ces rédies de la série 1 à la série 15 .

L'examen des stades parasitaires des rédies les plus matures observés chez les limnées de ces séries (fig. $3 \mathrm{~F}$ ) ne montre qu'un léger retard de maturation pour la série 15 .

\section{Discussion}

L'examen histologique de limnées tronquées infestées chacune par un seul miracidium permet de déterminer le nombre de rédies qui se forme à partir d'un seul sporocyste dans l'organisme du mollusque hôte. Dans une note antérieure (Rondelaud et Barthe, 1978 a), nous avons présenté un premier schéma illustrant le développement de ces générations rédiennes avec mention de leurs nombres respectifs chez des limnées tronquées examinées au $49^{\mathrm{e}}$ jour post-exposition à $20^{\circ} \mathrm{C}$ : les chiffres indiqués étaient cependant imprécis en raison : a) du faible nombre de limnées étudiées lors de cette période ( 5 individus) ; b) du regroupement dans la génération 3 des rédies tardives de génération 2 et des rédies de génération 3 par suite de l'impossibilité de les identifier après leur émergence du corps des rédies mères. Le nouveau schéma de développement, que nous proposons sur la figure 4 , indique le nombre moyen de rédies pour chaque génération et chaque catégorie au $30^{\mathrm{e}}$ jour post-exposition à $20^{\circ} \mathrm{C}$ : ces valeurs proviennent des résultats obtenus chez les 398 limnées infestées des séries 1 à 8 . Nous remarquons :

- que le sporocyste produit en moyenne 9,7 rédies de génération 1 regroupant 8,2 rédies indépendantes (dont 1,9 en dégénérescence) et 1,5 rédies encore dépendantes ;

- que la première rédie de génération 1 produit en moyenne 15,6 rédies précoces de génération 2 regroupant 13,8 rédies indépendantes (dont 1,3 en dégénérescence) et 1,8 rédies encore dépendantes;

- que les autres rédies de génération 1 produisent en moyenne 2,5 rédies tardives de génération 2 tandis que les rédies précoces indépendantes de génération 2 forment 4,1 rédies de génération 3 . 
La comparaison de nos résultats avec les données bibliographiques (indiquées ci-après) doit tenir compte des faits suivants : a) Les critères structuraux permettant d'identifier les rédies mères et les rédies filles indépendantes manquent (Thomas, 1883 ; Kendall, 1965) ou sont en opposition : les rédies filles indépendantes sont plus grandes que les rédies mères (Saint-Guillain, 1968), ou bien plus petites (Rondelaud, $1974 a$ ) avec un intestin plus court (Czapski, 1977); (b) Les rédies filles ont été identifiées dans le corps des rédies mères, parfois très tôt (Roberts, 1950), le plus souvent en présence de cercaires en formation bien reconnaissables (Chowaniec, 1961; Kendall, 1964 ; Hodasi, 1972 ; Wilson et Draskau, 1976). Elles correspondent donc à l'une ou à l'autre de nos générations rédiennes selon la période d'observation : ainsi les rédies filles étudiées par Wilson et Draskau au $30^{\circ}$ jour post-exposition à $20^{\circ} \mathrm{C}$ pourraient correspondre à nos rédies tardives de génération 2 , à celles de génération 3 et peut-être aux rédies précoces encore contenues dans la rédie no 1 de génération 1 .

Nos résultats confirment les données de Wilson et Draskau (1976) sur le nombre moyen de rédies indépendantes (22 rédies : fig. 4 contre 23 rédies) présenté par les limnées infestées individuellement par un seul miracidium et élevées à $20^{\circ} \mathrm{C}$ jusqu'au $30^{\mathrm{e}}$ jour post-exposition; de même le nombre moyen de rédies indépendantes et dépendantes obtenu dans ce travail, soit 32,2 rédies par limnée : figure 4 (valeurs extrêmes : 13-43 rédies) s'inscrit dans la gamme des valeurs rapportées par Kendall (1949), soit de 10 à 41 rédies par mollusque, chez des limnées infestées dans les mêmes conditions et sacrifiées entre le $44^{\mathrm{e}}$ et le $69^{\mathrm{e}}$ jours post-exposition. En revanche les données divergent :

- sur le nombre de rédies de génération 1 produites par un sporocyste : 9,7 rédies (fig. 4) contre 12-15 rédies pour Mattes (1949), 28 rédies pour Wilson et Draskau (1976) ou une seule rédie pour Czapski (1977). La discordance relevée entre nos valeurs et celles de Mattes ou de Wilson et Draskau peut s'expliquer par la terminologie utilisée : les rédies mères, que ces auteurs considèrent comme issues d'un seul sporocyste, peuvent correspondre à nos rédies indépendantes des générations 1 et 2 . Pour Czapski (1977), chaque sporocyste ne fournit qu'une seule rédie de génération 1 produisant elle-même les rédies filles de génération 2 ; le schéma proposé par cet auteur ne peut s'appliquer dans le cadre de notre étude en raison des deux observations suivantes : 1) le nombre maximum des rédies précoces de génération 2 formées par la rédie $n^{0} 1$ de génération 1 est de 19 (nombre moyen : 15,6 rédies) alors qu'au $30^{\mathrm{e}}$ jour post-exposition, les rédies indépendantes sont déjà 22 en nombre : figure 4 ; 2) si la rédie no 1 de génération 1 dégénère sans produire de rédies de génération 2 (une limnée sur 398), les rédies indépendantes présentant des cercaires en formation sont alors peu nombreuses au $30^{\mathrm{e}}$ jour post-exposition (8 dans ce cas) et présentent toutes un pharynx caractéristique de la génération 1 .

- sur le nombre de rédies tardives de génération 2 et de rédies de génération 3. En admettant que ces dernières rédies correspondent aux rédies filles indiquées dans la littérature, nos valeurs (6,6 rédies en moyenne par limnée : fig. 4) sont plus élevées que celles de Wilson et Draskau (2 rédies filles pour 50 rédies mères à $20^{\circ} \mathrm{C}$ ). 


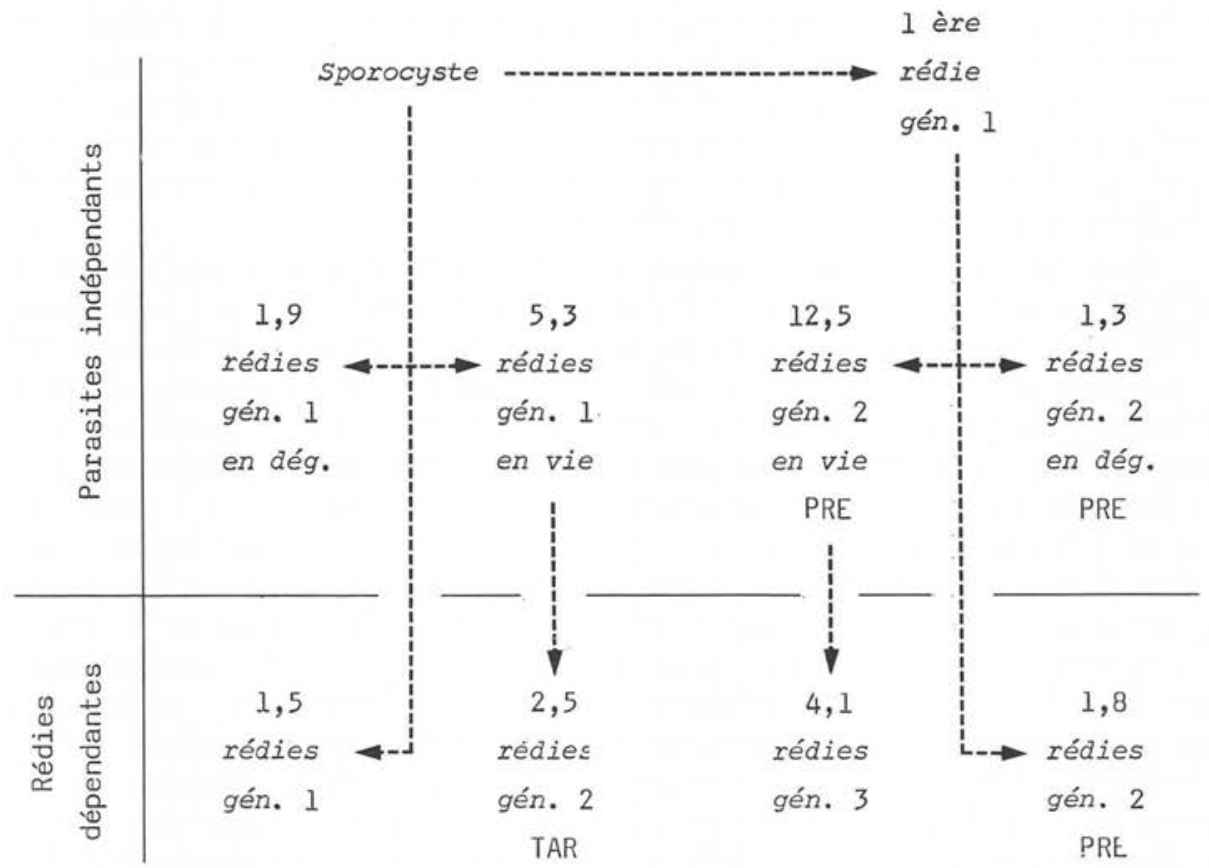

FIG. 4. - Schéma récapitulant les nombres de rédies formées à partir d'un seul sporocyste chez les 398 limnées des séries I à 8 (au $30^{\mathrm{e}}$ jour post-exposition à $20^{\circ} \mathrm{C}$ ).

gén. : génération; dég. : dégénérescence; PRE : précoces; TAR : tardives.

Cette discordance s'explique par le nombre de miracidiums utilisé par ces auteurs (5 miracidiums par limnée). La discordance disparaît lorsqu'on compare les valeurs rapportées par ces auteurs avec celles fournies par les limnées de la série 10 (5 miracidiums par exposition : fig. $2 \mathrm{E}$ ).

En accord avec Czapski (1977, 1978), il importe d'abandonner les termes de " rédie mère " et de " rédie fille " pour ne parler que de "génération rédienne ". Ce terme de génération rédienne a d'ailleurs été utilisé pour d'autres Fasciolidae comme Fasciola gigantica (Dinnik et Dinnik, 1956, 1964) et $F$. halli (Sinitsin, 1933). Ce terme doit cependant être nuancé dans son utilisation, puisque chaque poussée de rédies filles chez la limnée infestée comprend en réalité des rédies qui appartiennent à deux générations successives.

Notre étude expérimentale montre de plus que le schéma général d'évolution des générations rédiennes de $F$. hepatica est respecté dans le cas d'une infestation monomiracidienne individuelle. L'origine géographique du parasite n'a pas d'influence dans le cadre de cette étude. En revanche l'origine géographique des limnées d'expérimentation - et par suite la vitesse de croissance des individus de chaque population - n'influe que sur le nombre des rédies tardives de génération 2 et celui des 
rédies de génération 3. Ce dernier résultat confirme nos observations de 1978 (Rondelaud et Barthe, $1978 a$ et $b$ ) et montre l'importance du volume corporel du mollusque hôte dans le développement du parasite. Le rôle de ce facteur se retrouve d'ailleurs chez d'autres mollusques tels Biomphalaria glabrata ou Stagnicola palustris lorsqu'ils sont infestés respectivement par Schistosoma mansoni (Chernin et Prentice, 1978) ou Echinostoma revolutum (Zischke, 1967, 1968).

Une infestation plurimiracidienne perturbe le schéma de développement de ces générations rédiennes. Avec une seule exposition, les limnées soumises à des nombres élevés de miracidiums (de 5 à 20) présentent au 30e jour post-exposition : a) une réduction nette de leur nombre de rédies indépendantes : cette variation numérique retentit essentiellement sur les rédies précoces de génération 2 ; la génération 1 par contre ne varie guère en nombre, mais comprend un nombre assez élevé de rédies $n^{\circ} 1$; b) une augmentation sensible du nombre des rédies contenues dans les sporocystes ou les rédies no 1 de génération 1 ; c) la diminution, puis la disparition des rédies tardives de génération 2 et des rédies de génération 3. Les rédies indépendantes présentent d'autre part un retard de maturation d'autant plus grand que le nombre de miracidiums utilisé lors de l'exposition est plus élevé : ce retard peut s'expliquer par les notes de Kendall et Ollerenshaw (1963) démontrant qu'une augmentation du nombre des rédies dans le mollusque hôte est associé à une réduction du nombre de cercaires formées. Des résultats voisins s'observent lorsque la limnée subit 4 expositions monomiracidiennes successives. Toutes ces données sous-entendent que le volume corporel du mollusque hôte ne peut assurer le développement que d'un nombre déterminé de parasite (soit 1 à 2 miracidiums dans le cadre de cette étude).

Chez ces limnées à infestation plurimiracidienne, le nombre de sporocystes retrouvé au $30^{\mathrm{e}}$ jour post-exposition est toujours plus faible que le nombre de miracidiums utilisé lors de l'exposition. Deux hypothèses peuvent expliquer cette constatation : 1) tous les miracidiums ont disparu du récipient d'expérience lorsque la limnée en est retirée. Certains miracidiums n'auraient donc pas pénétré dans le mollusque hôte bien qu'ils soient accolés à l'épithélium tégumentaire de ce dernier ; 2) tous les miracidiums auraient pénétré dans le mollusque, mais un certain nombre d'entre eux dégénèrerait et disparaîtrait rapidement sans laisser de vestiges visibles dans l'organisme du mollusque. Seul un contrôle histologique des limnées présentant une infestation fasciolienne abortive pouıra apporter une solution à ce problème.

\section{BIBLIOGRAPHIE}

Chowaniec W. : Wplyw srodowiska na rozwoj form larwalnych motylicy watrobowej. - Fasciola hepatica oras zagadnienie superinwazji i reinwazji w zywicielu posrednim. Wiad. Parazytol., I96I, 7, 27I-273.

Christie J. D., Prentice M. A. : The relationship between numbers of Schistosoma mansoni daughter sporocysts and miracidia. Ann. Trop. Med. Parasitol., 1978, 72, 197-I98.

Czapsкi Z. : Biologiczne aspekty epidemiologii fasciolozy. Monogr. Podr. Skr. AWF w Poznaniu,

I977, 95, I-I 43.
CzApski Z. : New observations on the life-cycle of Fasciola hepatica L. in Galba truncatula O. F. Müll., Galba occulta Jack. and Galba turricula Held. I.C.O.P.A. IV, Short Communications, Varsovie, 1978, $A$, ro. 
Dinnik J. A., Dinnik N. N. : Observations on the succession of redial generations of Fasciola gigantica Cobbold in a snail host. Z. Tropenmed. Parasitol., 1956, 7, 397-419.

Dinnik J. A., Dinnik N. N. : The influence of temperature on the succession of redial and cercarial generations of Fasciola gigantica in a snail host. Parasitol., 1964, 54, 59-65.

Hodasi J. K. M. : The effects of Fasciola hepatica on Lymnaea truncatula. Parasitol., 1972, 65, 359-369.

KENDALL S. B. : Nutritional factors affecting the rate of development of Fasciola hepatica in Limnaea truncatula. J. Helminthol., I949, 23, I79-190.

KENDALL S. B. : Some factors influencing the development and behaviour of trematodes in their molluscan hosts. In : "Host-parasite relationships in invertebrate hosts " par A. E. R. Taylor, pp. 51-93. Blackwell, Oxford, r964.

Kendal. S. B. : Relationships between the species of Fasciola and their molluscan hosts. Adv. Parasitol., $1965,3,59-98$.

Kendall S. B., Ollerenshaw C. B. : The effect of nutrition on the growth of Fasciola hepatica in its snail host. Proc. Nutr. Soc., 1963, 22, 4I-46.

Leuckart R. : Zur Entwicklunggeschichte des Leberegels. Zweite Mitteilung. Zool. Anz., I882, S, 524-528.

LeUCKART R. : Zur Entwicklunggeschichte des Leberegels (Distomum hepaticum). Arch. Naturgeschichte, $1883,48,80-119$.

MatTEs O. : Wirtsfindung, Invasionsvorgang und Wirtsspezifität beim Fasciola-Miracidium. Z. Parasitenkd., 1949, $14,320-363$.

Ollerenshaw C. B. : Some observations on the epidemiology of fascioliasis in relation to the timing of molluscicide applications in the control of the disease. Vet. Rec., I971, 98, I52-I64.

Roberts E. W. : Studies on the life-cycle of Fasciola hepatica (Linnaeus) and of its snail host, Limnaea (Galba) truncatula Müller in the field and under controlled conditions. Ann. Trop. Med. Parasitol., 1950, 44, 187-206.

Rondelaud D. : L'évolution des rédies de Fasciola hepatica L. chez Galba truncatula Müller en Limousin. Rev. Méd. Vét., I974 $a, 125,237^{-250}$.

RoNDElaud D. : Recherches sur l'influence de quelques facteurs physiques sur les migrations quotidiennes de Galba truncatula Müller. Ann. Parasitol. Hum. Comp., 1974 b, 49, 41 7-425,

RoNDELAUD D. : The redial generations of Fasciola hepatica L. in Lymnaea (Galba) truncatula Muller. I.C.O.P.A.IV, Short Communications, Varsovie, 1978, C, 85 .

RonDelaud D., BARThe D. : Arguments et propositions pour une nouvelle interprétation de l'évolution de Fasciola hepatica L. dans Lymnaea (Galba) truncatula Müller. Ann. Parasitol. Hum. Comp., I978 $a, 53,201-2 \mathrm{I} 3$.

Rondelaud D., Barthe D. : Êtude histologique du développement de Fasciola hepatica chez Lymnaea truncatula, L. glabra et $L$. palustris infestées dès leur naissance. C.R. Soc. biol., $1978 b, 172$, I $194^{-1} 200$.

Rondelaud D., BARThe D. : Fasciola hepatica L. : Les formes larvaires non évolutives ou en dégénérescence chez Lymnaea truncatula Müller. $Z$. Parasitenkd., 1980, 62, 95-104.

Saint-Guillain M. : Étude histologique des premiers stades évolutifs de Fasciola hepatica L. Acta Zool. Pathol. Antwerp., 1968, 46, 77-132.

Sinitsin D. F. : Studien über die Phylogenie der Trematoden. Z. Parasitenkd., 1933, 6, I70-I9I.

STYCZYNSKA-JuREWICZ E. : Adaptation of eggs and larvae of Fasciola hepatica to the conditions of astatic habitats of Galba truncaluta. Acta Parasitol. Polon., I965, I3, I5I-I70.

Thomas A. P. : The life history of the liver fluke (Fasciola hepatica). Quart. J. Micr. Sci., I883,

WILSON R. A., DRASKAU T. : The stimulation of daughter redia production during the larval development of Fasciola hepatica. Parasitol., 1976, 72, 245-257.

ZISchKE J. A. : Redial populations of Echinostoma revolutum developing in snails of different sizes. J. Parasitol., 1967, S3, 1200-1204.

ZISchKe J. A. : Reproduction of Echinostoma revolutum rediae when transferred to uninfected snails of different sizes. J. Parasitol., 1968, 54, 39-42. 